\title{
Bioactive Compounds of Garlic: Role in The Management of Various Types of Cancer
}

\author{
Saleh A. Almatroodi *,a \\ a Department of Medical Laboratories, College of Applied Medical Sciences, Qassim University, Buraydah, \\ Saudi Arabia
}

\begin{abstract}
Allium Sativum L., well known as garlic, a member of the family Liliaceae, is one of the most commonly used spice and plant-based medicine worldwide. The health promoting effects of allicin, diallyl sulfide, diallyl disulfide and diallyl trisulfide, the principal active component of garlic has been established through its antioxidant potential. Moreover, bioactive compounds of garlic have shown their therapeutic implications in chemically induced carcinogenesis in animal models through suppression or inhibition of promotion and progression of carcinogenesis. Moreover, bioactive compound has revealed chemopreventive effect on various types of tumor including breast, liver, skin, pancreas, oral and prostate through inhibiting the cell proliferation. This review summarizes the mechanism of action and anti-cancerous effects of allicin, ajoene, diallyl sulfide, diallyl disulfide and diallyl trisulfide on different types of cancer. Further studies based on animal model are warranted to evaluate the efficacy, safety and mechanism of action of bioactive compound in tumor management.
\end{abstract}

Keywords: Allicin, Diallyl sulfide, Diallyl disulfide, Cancer

Citation: Almatroodi, S.A. Bioactive Compounds of Garlic: Role in The Management of Various Types of Cancer. PHARMANEST Int J Adv Pharm Sci 2020; 11(1): 1-13. doi: 10.46424/pharmanest.11.1.2020.1-13.

(C) Copyright 2020

This is an open access article distributed under the terms of the Creative Commons Attribution License (CC BY 4.0), which permits unrestricted use, distribution, and reproduction in any medium, provided the original author and source are credited.

\section{Introduction}

Cancer is notorious killer disease and major health concern worldwide. Though, the major concern regarding commonly used cancer drugs are they cause toxicity, adverse effects on normal cells and also alter the various cell signaling pathways. Moreover, commonly used mode of treatment such as radiotherapy caused various side effects including mucositis, oral candidiasis and loss of taste, that may be permanent due to the destructive effect of radiation on the salivary glands (1). In the treatment

\footnotetext{
* Corresponding author: Saleh A. Almatroodi (smtrody@qu.edu.sa)
}

of cancer, natural products or active compound of medicinal plants signify alternative solutions to current medicine through killing of cancer cells or inhibiting the growth of cancer cells. In this regard, garlic (Allium sativum L. family Liliaceae) has a long history of use in the disease due to their anti-inflammatory, anti-tumor and antioxidant properties. On the other side, garlic is mixture of various compound such as allicin and diallyl disulfide and such compounds are key player in the diseases management without causing any adverse effects on the physiological as well as biochemical process. However, it has been proven that garlic and their ingredients are modulator of cell signaling pathways and stimulator of several physiological 
cascades and finally causes cancer growth inhibition. A pioneer study supports the role of garlic in cancer as diallyl sulfide, diallyl disulfide and garlic extract showed role in reduction of anti-proliferative gene and propose that modulation of apoptosislinked cellular proteins in the in non-small cell lung cancer cells (2). Besides, other finding demonstrated that diallyl trisulfide, active compound of garlic has proven anti-cancer effects through the promotion of apoptosis and fresh garlic juice induces apoptosis via increasing caspase- 3 activity and Bax: $\mathrm{Bcl} 2$ ratio in the oral squamous cell carcinoma (3). Garlic extract showed role in the inhibition of growth and induction of apoptosis via the inhibition of the PI3K/Akt pathway, demonstrating that extract might be powerful therapeutic agent in the prevention and treatment of colon cancer (4). In the support of garlic role in human colorectal cancer cells, study reported that garlic extract reduced cell cycle progression via downregulating cyclin B1 and cdk1 expression through inactivation of NF-kB (5).

\section{Methodology}

A wide-ranging literature search was carried out through surveying appropriate peer-reviewed research/review articles using databases including PubMed, ResearchGate, Science Direct, Web of science, Scopus, and Google Scholar, for studying the implication of active compound of garlic in the management of cancer. The keywords were used to complete the study was allicin, diallyl sulfide, diallyl disulfide, cell signaling pathways, different types of cancer, in vitro and in vivo study. Inclusion criteria were peer reviewed journal articles, conference/ seminar proceedings and refereed books. Emphasis was given to search the therapeutic role of bioactive compound of garlic in the management of various types of cancer through modulating cell signaling pathways. Non-English language articles and thesis/dissertation were not included in the study. Finally, 104 references have been used to complete the study with the literature cited up to 2019.

\section{Possible mechanisms of action of garlic and their active compounds}

The possible mechanisms of action of garlic and their active ingredient in the management of cancer growth are described as;

3.1. Tumor angiogenesis is one of the vital process in cancer formation and its inhibition is crucial step in the tumor growth inhibition. Evidences advocate that garlic and its bioactive compound illustrations its anti-cancer activity by inhibiting the angiogenesis process. In this regards, previous study reported that allicin, an active compound of garlic significantly inhibited fibroblast growth factor-2 and vascular endothelial growth factor secretion in a concentration-dependent manner (6).

3.2. Earlier studies evidence that garlic and its active compound play an important role in the inhibition of cancer cell growth through causing cell cycle arrest at the G0/G1and $G_{2} / M$. In this support, previous finding demonstrated that diallyl disulfide meaningfully inhibited the cell proliferation, induced G2/M cell arrest and prolong the cell cycle by inducing $\mathrm{G} 2 / \mathrm{M}$ arrest to inhibit proliferation of colon cancer cells (7).

3.3. PTEN is a negative regulator of a cell growth and survival signaling pathway, that is the phosphatidylinositol-3-kinase (PI3K)/AKT signaling pathway $(8,9)$. In this regard, it was proven that garlic extract showed role in the regulation of the function of the PI3K/Akt pathway through upregulating PTEN (4).

3.4. Herbs or its active has proven their role in cancer management through induction of apoptosis. However, garlic also confirmed its role in cancer inhibition through induction of apoptosis. It was reported that S-allylmercaptocysteine, an active compound of garlic induces apoptosis through JNK and p38 signaling pathways, increasing tumor protein p53 and Bax activation in the SW620 cells (10).

\section{Garlic and its bioactive compounds: Role in management of different types of cancers}

Garlic and their bioactive compounds play a significant role in management of various types of cancer [ Figure 1]. It employs suppressive role on tumor formation and oxidative damage induced free radical agents. Moreover, garlic and their active compound shows its anti-cancerous activity via modulating the various potential targets like apoptosis, angiogenesis, tumor suppressor genes, Transcription factors and another cell signaling pathways.

\section{The role of bioactive components of garlic in killing of cancer cells and inhibition of growth of tumor is explained in detailed as:}

\subsection{Breast cancer}




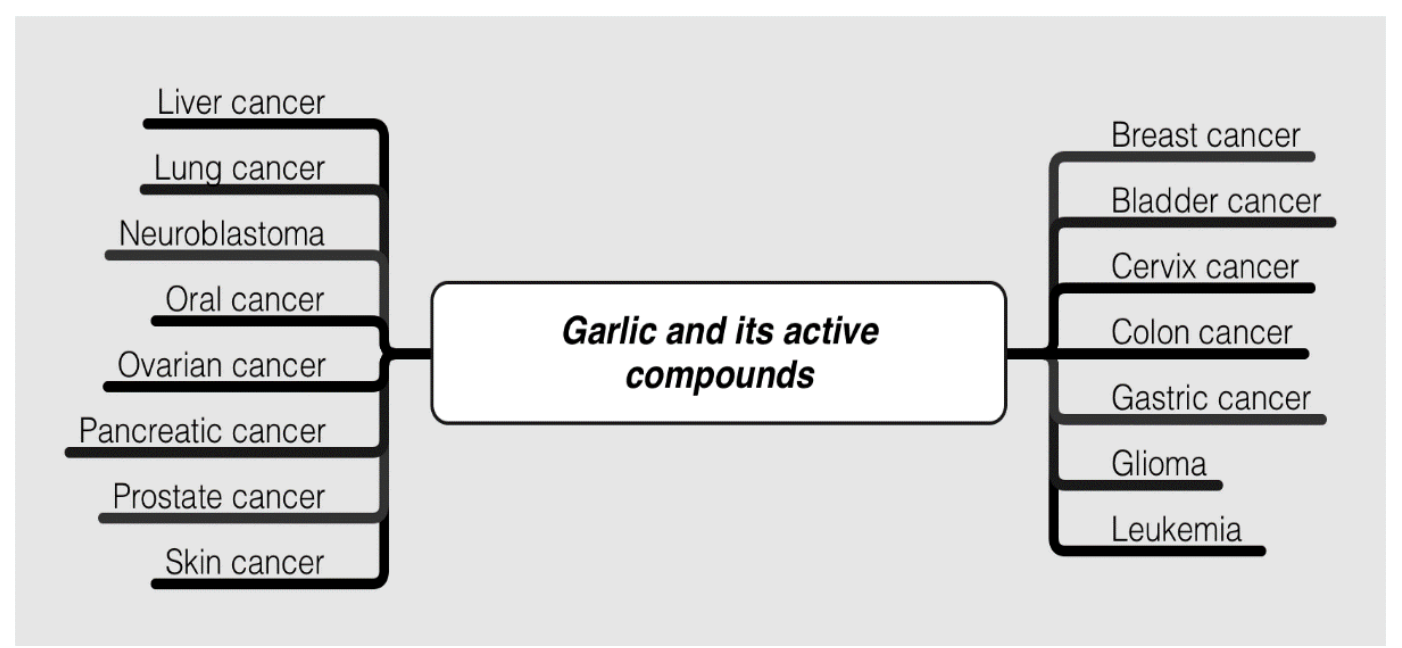

Figure 1. Garlic and its active compound shows role in the inhibition of various types of cancer.

Breast cancer is one of the most common cancer in term of morbidity and mortality. The chemotherapeutic drugs used in the treatment of breast cancer is effective but cause adverse effect on normal cells and also alter physiological process. However, in this regard, garlic and their active constituent has proven chemopreventive effect without any adverse effect on normal cells and promote physiological process. Allicin, active ingredient of garlic, significantly reduced cell migration and invasion in MCF-7 cells. Moreover, protein interaction between ER- $\alpha$ and p65 was down-regulated via TNF- $\alpha$ treatment, and such effect was reversed by allicin and allicin powerfully suppressed TNF- $\alpha$-induced activation of ERK1/2 (11). Another pioneer study reported that diallyl disulfide, an ingredient of garlic synergizes the effect of breast cancer suppressor such as eicosapentaenoic acid, and antagonizes the effect of breast cancer enhancer such as linoleic acid. Consequently, garlic and garlicderived compounds are promising candidates in the breast cancer control (12). Furthermore, other results propose that diallyl disulfide showed role in the inhibition of the proliferation of cancer cells including MCF-7 cells as well as induction of apoptosis of cancer cells and mechanisms may comprise the inhibition of ERK and the activation of the SAPK/JNK and p38 pathways (13). Another study based on breast cancer cells line including MDA-MB-231 cells was performed. Diallyl disulfide antagonized the effect of linoleic acid, a potent breast cancer cell enhancer and synergized the effect of eicosapentaenoic acid), a potent breast cancer cell suppressor (14). Histone deacetylation inhibitors are recognised its role in the suppression of cancer growth as well as induction of apoptosis in cancer cells and properties of diallyl disulfide may be accountable in the induction of apoptosis in breast cancer cells (15) and diallyl trisulfide has confirmed role as anti-proliferative in human breast cancer cells lines (16). Similarly, Diallyl disulfide effects on breast cancer cells was examined and finding confirmed that active compound of garlic inhibited the proliferation of cancer cells in a duration- and dose-concentration manner. Furthermore, both migration and invasion were significantly inhibited upon exposure to different concentrations of diallyl disulfide (17). Antitumor effect of diallyl disulfide on triple-negative breast cancer cells is mediated by the $\beta$-catenin pathway, proposing that diallyl disulfide might be used as a potential therapeutic agent in the treatment or prevention of breast cancer (18). Diallyl trisulfide, active compound of garlic showed role in the suppression of breast cancer stem cells via inhibition of $\mathrm{Wnt} / \beta$-catenin pathway activation (19). Oral treatment of mice with concentration of $20 \mu \mathrm{mol}$ DATS earlier to challenge with a carcinogen such as benzo[a]pyrene caused around $85 \%$ decrease in forestomach tumor multiplicity (20).

\subsection{Brain cancer}

The anticancer activity of Allicin on human glioma cells and its principal mechanism was examined. Results of the study advocate that allicin suppresses proliferation and induces glioma cell apoptosis based in vitro experimentation. Moreover, both intrinsic mitochondrial and extrinsic Fas/FasL\&\#8209;mediated pathways react in glioma cell after treating with allicin, which then activate chief apoptotic cascades (21). Effect of allicin on the cell viability of U87MG human glioma cells was examined. Result showed that allicin inhibited the cell viability of U87MG human glioma cells in a doseand time-dependent manner and allicin-induced inhibition of cell viability was due to apoptosis of 
cells (22). Previous fining based on result concluded that allicin, a compound of garlic can improve $T$ cell subsets distribution and inhibit VEGF expression via its immunomodulatory activity, thus improve the efficacy on neuroblastoma in coordination with cyclophosphamide (23). A study was made to assess the ability of S-allyl cysteine (SAC), to affect the proliferation and differentiation of human neuroblastoma cells in vitro. Result demonstrated that time-and dose-dependent inhibition of cell grow was observed in cultures treated with SAC and results indicate that SAC can inhibit human neuroblastoma cell growth in vitro (24). Treatment of neuroblastoma cell with DADS caused arrest of cell cycle in G (2)/M phase and commitment to apoptosis via the activation of the mitochondrial pathway $\mathrm{Bcl}-2$ down-regulation, cytochrome c release into the cytosol, and activation of caspase-9 and caspase-3 (25). Diallyl trisulfide suppresses survival, migration, invasion and angiogenesis in glioma cells and such effects were associated with inhibition of the $\mathrm{Wnt} / \beta$-catenin signaling cascade (26).

\subsection{Bone cancer}

Diallyl trisulfide, active compound of garlic showed role in the suppression of cell survival, woundhealing capacity, invasion and angiogenesis based on osteosarcoma. cells Additionally, such effects were linked with decreased expression of Notch-1 and its downstream genes, such as vascular endothelial growth factor and matrix metalloproteinases. Finally, this study based on finding advocate that diallyl trisulfide inhibited osteosarcoma growth and aggressiveness (27). Diallyl disulfide treatment caused noteworthy downregulation of cyclin D1, c-myc, and lymphoid enhancer-binding factor 1 expression, whereas remarkably upregulated p21 level in U2OS cells. Diallyl disulfide might be a promising anticancer agent for Osteosarcoma, and the fundamental mechanisms might be linked with the anti-proliferation and anti-invasion properties (28). Recent study based on active compound of garlic including diallyl trisulfide was performed to check its efficacy and reported that exposure to diallyl trisulfide change the morphology and inhibited the growth of the human osteosarcoma cells such as Saos-2 cells, and its effects was concentration- and exposure time-dependent (29). Osteosarcoma based study reported that diallyl trisulfide showed a vital role in the reduction of cell viability in a doseand time-dependent manner in osteosarcoma cells. In addition, diallyl trisulfide -induced G0/G1 phase arrest was established to associate with a decrease in cyclin D1 in concomitance with an increase in p21 and p27 (30). The viability of osteosarcoma cells in the artesunate and allicin group was significantly decreased in a concentration and time dependent manner; likewise, invasion, motility and colony formation ability were significantly suppressed and the apoptotic rate was significantly increased (31).

\subsection{Cervix cancer}

Cervical cancer is notorious killer and has high incidence in developing world. The current mode of treatment based on radiotherapy and chemotherapy are expensive and also alter the physiological process. However, inexpensive and safe mode of treatment is needed to overcome such problem. In this regard, medicinal plants based products play a vital role due to its rich source of antioxidant and scavenge free radicals and inhibit genetic damage.

Anti-cancer effects of diallyl sulfide in human cervical cancer cells was examined and the underlying mechanisms in vitro was also investigated. Outcome of the study showed that diallyl sulfide treatment noticeable decrease in cell viability time- and dosedependently. Moreover, diallyl sulfide induced G0/G1 cell cycle arrest and sub-G1 phase and cells treated with different concentrations of diallyl sulfide also showed changes in apoptosis such as morphological changes (32). Recent study based on cervical cancer was made and result demonstrated that diallyl disulfide induced apoptosis through the production of reactive oxygen species, and induced abrogation of mitochondrial membrane potential. Diallyl disulfide increased the levels of p53, p21 and Bax, but caused a decrease in the level of Bcl-2 (33). Another study result reported that diallyl disulfide in HeLa cells exposed to radiation resulted in decrease in cell viability. Moreover, cells pre-treated with diallyl disulfide clearly inhibited the radiation-induced G2/M phase arrest, whereas promoted radiation-induced apoptosis (34). Pioneer study was performed based on cervical cancer cells and finding revealed that allicin was effective in suppressing the malignant phenotype of cervical cancer cells mainly through inhibiting the expression of NRF2 (35).

\subsection{Colon cancer}

Several natural products or active compound of herbs has shown their role in the inhibition of colon cancer through killing of cancer cells or inhibition of growth of cancer. S-allylcysteine and Sallylmercaptocysteine water soluble compound of garlic was examined for their role on proliferation and cell cycle progression based on colon 
cancer cell lines. Outcome demonstrated that Sallylmercaptocysteine, whereas not S-allylcysteine, inhibited the growth of cell lines at doses similar to that of sulindac sulfide. S-allylmercaptocysteine also showed role in the induction of apoptosis, and this was linked with an increase in caspase3-like activity (36). Garlic compound such as diallyl disulfide on human colon cancer cell line on cell cycle arrest and apoptosis was performed to evaluate its role. After the treatment of cancer cells with diallyl disulfide, the dose- and time-dependent decreases of viable cells were noticed. In addition, the treatment of colon cancer cells with diallyl disulfide resulted in G2/M phase arrest and apoptosis incident by the mitochondrial-pathway (37). Anticancer effect of garlic by using human colon cancer cell lines was examined. The growth of the cells was significantly suppressed by diallyl trisulfide, additionally, the proportion of used cancer cells residing at the $G_{1}$ and $S$ phases were decreased by diallyl trisulfide, and their populations at the $G_{2} / M$ phase were obviously increased (38). Previous study was performed to examine the role and the molecular mechanism of diallyl disulfide in the induction of cell cycle arrest based on human colon cancer cell line. Result of the study proved that diallyl disulfide significantly inhibited proliferation and retarded the population doubling time of cancer cells. Furthermore, diallyl disulfide significantly decreased the protein contents of p53 and cyclin B1, whereas increased the expression of p21WAF1 in a time and dose-dependent manner (7). Anti-proliferative activity of diallyl disulfide and screening of differentially expressed genes induced by diallyl disulfide was examined. Result of the study confirmed that diallyl disulfide showed anti-proliferative effects on colon cancer cells (39). Cytotoxic effects in diallyl trisulfide on colorectal cancer cells was investigated. Result demonstrated that diallyl trisulfide inhibited the viability of primary colorectal cancer cells in a time- and dose-dependent manner. After treatment with diallyl trisulfide, colorectal cancer cells exhibited condensation of DNA and it increased reactive oxygen species production in colorectal cancer cells (40). Study finding revealed that levels of colorectal cancer stem cells markers elevated in the tumorspheres cells. As well, diallyl trisulfide powerfully suppressed the activity of colorectal cancer stem cells, as recognised by reducing the size and number of colonspheres, decreasing the expression of colorectal cancer stem cells markers (41). Another study reported that diallyl sulfide when given to mice, inhibited by $74 \%$ the incidence and reduced the frequency of colorectal adenocarcinoma (42).

\subsection{Gastric cancer}

A recent study was performed using proteomic means, studied the responses of protein expression in gastric cancer cell line, caused by diallyl trisulfide. A distinctive protein in BGC823 cells were detected with noteworthy changes in their expression levels corresponding with diallyl trisulfide administration. Moreover, proteomic results offered additional support to the hypothesis that garlic is a strong inducer of apoptosis in tumor cells (43). Another study was performed to equate the protein expression profile of gastric cancer cells exposed to diallyl trisulfide treatment. Finding of the study revealed that downregulation of UPAR may partially be accountable for diallyl disulfide -induced inhibition of ERK/Fra-1 pathway, and cell migration and invasion (44). The role of diallyl trisulfide, ingredient of garlic, and docetaxel on regulation of MT2A in relation to NF- $k B$ in gastric cancer cells was examined. Result confirmed that diallyl trisulfide showed its anti-Gastric cancer activity and enhances chemosensitivity of gastric cancer to docetaxel via epigenetic upregulation of MT2A to attenuate NF- $\kappa$ B signaling (45). Treatment of human gastric cancer cells with diallyl trisulfide, compound of garlic in vitro and in vivo significantly activated kinases including p38 and JNK/MAPK and attenuated the Nrf2/Akt pathway and diallyl trisulfide exerts anticancer effects and improves the antitumor efficacy of cisplatin (46). Human gastric cancer cell line based study reported that growth of cancer cells was inhibited by diallyl disulfide. Cells treated with diallyl disulfide showed a lower nucleocytoplasmic ratio and inclined to form gland and intercellular conjunction structures (47). A pioneer study was aimed to explore the effects and the essential mechanism of allicin on gastric cancer cells. Result showed that allicin reduced cell viability in a dose- and time-dependent manner. Moreover, at the molecular level, allicin induced cytochrome $c$ release from the mitochondria and increased caspase- $3,-8$, and -9 activation, with concomitant upregulation of Bax and Fas expression in the tumor cells (48). The role of allicin on both telomerase activity and apoptosis in gastric cancer cells was performed. Result showed that allicin inhibited telomerase activity in a time-dependent and dose-dependent pattern (49). Allicin showed role in the inhibition of the proliferation and induction of the apoptosis of human gastric carcinoma cells, and this may partially be attained via the enhanced expression of p38 and cleaved caspase 3 (50). 


\subsection{Kidney cancer}

Renal cell carcinoma based study was performed and result explained that protein levels of $\mathrm{BCl}-2, \mathrm{VEGF}$ and HIF- $1 \alpha$ were increased in renal cell carcinoma tissues. Interestingly, treatment with allicin significantly decreased HIF-1 $\alpha$ protein level, so reducing Bcl-2 and VEGF expression and enhanced apoptotic cells (51).

\subsection{Liver cancer}

Experiment was performed to know the modes of mechanism of cell death caused by allicin, a chief ingredient of garlic based on human hepatoma cells. Based on finding result advocate that allicin caused cell death in human hepatoma cells via either autophagy or apoptosis (52). Allicin enhanced 5fluorouracil inducing cytotoxicity in hepatocellular carcinoma cells. In vivo experiment, combined treatment group with allicin and 5-fluorouracil showed an intense inhibitory effect on the growth of hepatocellular carcinoma xenograft tumors. The cotreatment group showed highly apoptotic level compared with 5-fluorouracil treated alone (53). Another study reported that diallyl trisulfide might affect cell viability and cell morphological changes in human liver tumor cells and lead cells to be arrested in G2/M phase via controlling the expression of cyclin B1 and Cdk7 in liver tumor cells (54).

\subsection{Leukemia}

In vivo effects of diallyl sulfide on leukemia cells was evaluated and finding revealed that diallyl sulfide decreased the percentage of viable cancer cells and these effects are dose-dependent and diallyl sulphide decreased the percentage of Mac-3 and CD11b, indicating that the differentiation of the precursor of macrophage cells was inhibited (55). Earlier instigator based on leukemia cells and leukomic mice was performed and result showed that diallyl trisulfide has proven its role in induction of cell death through induction of apoptosis in leukemia cells and diallyl trisulfide also promotes immune responses in leukemia and normal mice in vivo (56). Diallyl disulfide efficiently inhibit the proliferation and induce apoptosis of human leukemia cell line. Moreover, inhibition of ERK signaling pathways and activation of p38 signaling pathways are possibly involved in diallyl disulfide induced apoptosis in HL-60 cells (57). Suda and colleagues have explained that diallyl trisulfide treatment noticeably induced HSP27 protein in human monocytic leukemia cells (58) and treatment with diallyl trisulfide resulted in meaningfully inhibited leukemia cell growth in a concentration- and time-dependent manner via induction of apoptosis.
In U937 cells, DATS-induced apoptosis was correlated with down-regulation of $\mathrm{Bcl}-2$ and activation of caspases (59). Apoptosis in leukemia cells triggered through ajoene, a compound from garlic is based on the activation of a mitochondria-dependent caspase cascade which includes also the activation of the initiator caspase-8 (60).

\subsection{Lung cancer}

Antitumor effect of allicin, an active component of garlic against lung adenocarcinoma cells was examined and it was observed that cell proliferation was significantly reduced in cancer cells with treatment with allicin. Additionally, it was noticed that allicin treatment decreased the tumor cell adhesion to fibronectin in a concentration-dependent manner and number of migratory cells decreased in a dosedependent manner (61). Pioneer study based on lung cancer cells was performed and it was demonstrated that exposure of cancer cells to diallyl trisulfide showed significant improvement of G2-M fraction. Likewise, diallyl trisulfide-mediated G2-M phase cell cycle arrest in both used cancer cells was marked as initial as after treatment and convoyed by a decrease in primarily G0-G1 phase cells (62). Antitumor properties of diallyl trisulfide on lung cancer and synergistic effects of diallyl trisulfide combined with Cisplatin on the xenograft model was evaluated. Result of the study has confirmed the decreased cell viabilities, cell cycle $G_{1}$ arrest, and apoptosis induction were observed in diallyl trisulfide treated cancer cells. And injection of diallyl trisulfide to mice meaningfully inhibited the growth of human cancer cell tumor xenograft. In addition, diallyl trisulfide in combination with Cisplatin showed enhanced anti-tumor activity through induction of apoptosis (47). Similarly, another study reported that oral gavage of diallyl trisulfide, significantly retarded growth of A549 xenografts in nude mice without causing any negative side effects compared with control group. Also, the evidence both in vitro and in vivo advocated that diallyl trisulfide might be a novel anti-cancer drug (63). A population-based study was completed on Chinese population with the aim to search the association between raw garlic consumption and lung cancer. Based on finding study concluded that protective linked between eating of raw garlic and lung cancer has been noticed with a dose-response pattern (64). Another study was performed on the Chinese population to examine the association between raw garlic consumption and lung cancer. The results of the study demonstrated that raw garlic consumption is associated 
with reduced risk of lung cancer (65). Previous study was performed to examine the consumption of Sallylcysteine might prevent the growth of human non-small-cell lung carcinoma. It was observed that S-allylcysteine significantly inhibited the proliferation of cancer cell in vitro. Besides, result showed that S-allylcysteine significantly supressed the activation of molecules such as mTOR, NF-kB and cyclin D1 in vitro (66).

\subsection{Lymphoma}

The role of diallyl disulfide, compound of garlic, on telomerase activity in human lymphoma was executed. Result has shown that diallyl disulfide -mediated decrease in telomerase activity in cancer cells with concomitant transcriptional downregulation of human telomerase reverse transcriptase. Study advocate that diallyl disulfide downregulate telomerase activity through c-Myc-, Sp$1-$, and Mad1-dependent transcriptional downregulation of hTERT (67). Similarly, study based on primary effusion lymphoma reported that diallyl trisulfide suppressed the production of progeny virus from primary effusion lymphoma cells. The administration of diallyl trisulfide suppressed the growth of primary effusion lymphoma cells and these findings deliver confirmation that diallyl trisulfide has antitumor activity against primary effusion lymphoma cells in vitro and in vivo (68).

\subsection{Oral cancer}

Oral cancer is one of the principal causes of death worldwide. The current mode of treatment regarding oral cancer causes negative side effect including loss of taste and permanent damage of salivary glands. Compounds including curcumin, thymoquinone, oleuropein and metabolites derived from herbs play an important role in oral cancer management through modulating cell signaling pathways (69-73). Study was investigated to check the S-allylcysteine consumption role in prevention of tumor growth and progression in a mouse xenograft model of oral cancer. Finding demonstrated that Sallylcysteine dose-dependently inhibited the growth of cancer in tumor-bearing mice. S-allylcysteine was able to effectively suppress the tumor growth and progression in vivo and S-allylcysteine inhibited the expression of genes including cyclo-oxygenase-2, vimentin and NF- $\kappa$ B p 65 (74). Apoptotic effects of garlic fresh juice on the squamous cell carcinoma cells was evaluated and result of the study concluded that juice induces apoptosis in the cancer cells via increasing caspase-3 activity (3).

\subsection{Ovarian cancer}

Several types of active compounds of medicinal plant have confirmed their role as chemopreventive through their ability to kill cancer cells. Sallylcysteine, a water-soluble garlic derivative, on human ovarian cancer cells was evaluated. Study finding exhibited that treatment of cancer cells with S-allylcysteine resulted in $G_{1} / S$ phase arrest and induced apoptosis (75). Epigenetic mechanism of S-allyl cysteine was examined and it was reported that treatment of ovarian cancer cell with S-allylcysteine caused in G1/S phase arrest and decreased DNA methylation levels in cancer cells in a dose-dependent manner. Also, Sallylcysteine treatment showed re-expression of the mRNA and proteins of silenced tumor suppressor gene CDKN1A (76). The apoptosis rates of ovarian cancer cells were increased in diallyl trisulfide groups when compared with control, with dosedependence, the mRNA and protein expressions of PUMA, Bax was upregulated whereas $\mathrm{BCl}-2$ were down-regulated in diallyl trisulfide groups (77). The apoptotic pathways induced by allicin in the human ovarian cancer cell line was investigated and result has shown that allicin induced cancer cell apoptosis and JNK phosphorylation in a time- and dosedependent manner (78).

\subsection{Pancreatic cancer}

The efficacy of treatment with recombinant interleukin-2 and allicin as combination on pancreatic cancer was evaluated. Result proven that treatment with allicin and recombinant interleukin-2 resulted in suppression of tumor growth and prolonged survival time (79). The role of diallyl trisulfide on pancreatic cancer cells was investigated. Outcome of the study exhibited that diallyl trisulfide suppressed the viability of cultured human pancreatic cancer cells via increasing the proportion of cells in the G2/M phase and induced apoptotic cell death (80). Efficacy of diallyl trisulfide against pancreatic cancer cells was evaluated. Finding revealed that treatment with diallyl trisulfide inhibited the growth of pancreatic cancer cells in a dose-dependent manner and induced apoptosis in pancreatic cancer cells (81) and finding advocate that diallyl trisulfide anti-cancer activity through inhibition of growth of cancer cells.

\subsection{Prostate cancer}

Diallyl disulfide, an active ingredient of garlic showed pivotal role in the inhibition of the growth of prostate cancer cells in a dose dependent manner and this 
compound induced DNA damage at certain concentrations. Additionally, it was established that diallyl disulfide, inhibits proliferation of prostate cancer cells via the induction of apoptosis (82) and diallyl disulfide inhibited the growth of prostate cancer cells in a dose dependent manner and induced cell cycle arrest at G2/M transition in cancer cells (83). Diallyl trisulfide study based on prostate cancer cells showed role in the reduction of androgen receptor mRNA level, protein level, transcriptional activity, and PSA secretion (84). Earlier study based on prostate cancer reported that diallyl disulfide reduced the secretory activity with the slow increase in dosage and garlic compound such as diallyl disulfide induced apoptosis and nuclear segmentation at the higher doses (85). In this regard, another study demonstrated that diallyl trisulfide-induced apoptosis in cancer cells was linked with phosphorylation of $\mathrm{BCl}-2$, reduced $\mathrm{Bcl}-2$ : Bax interaction, and cleavage of procaspase-9 and -3. Bcl-2 overexpressing PC-3 cells were meaningfully more resistant to apoptosis induction by diallyl trisulfide (86). Mitochondria-mediated cell death by diallyl trisulfide is associated with reactive oxygen species generation and regulated by Bax/Bak and this finding was based on prostate cancer cells (87). Active compound of garlic such as diallyl trisulfide showed that it inactivates Akt to trigger apoptosis. Moreover, treatment of cancer cells including PC-3/DU145 cells with apoptosis including concentration of diallyl trisulfide caused in a rapid decrease in Ser 473 and $\mathrm{Thr}^{308}$ phosphorylation of Akt leading to inhibition of its kinase activity (88). Study was performed to compare the cytotoxic effects of diallyl trisulfide on prostate cancer cells and noncancerous human prostate epithelial cells. Result demonstrated that noncancerous human prostate epithelial cells had higher resistance to diallyl trisulfide induced cell death than prostate cancer cells and cytotoxicity of diallyl trisulfide toward noncancerous human prostate epithelial cells was strongly reduced as opposed to cancer cells (89). Fluorescent orthotopic androgen-independent prostate cancer mouse model was established to evaluate the potentiality of S-allylmercaptocysteine to inhibit tumor growth. Finding revealed that S-allylmercaptocysteine inhibited the growth of primary tumors and reduced the number of lung and adrenal metastases (90).

\subsection{Skin cancer}

There are various reports are documented in the support of natural products or active compound of plants in the treatment of skin diseases. Besides, phenolic compounds are rich source of antioxidant and that compound are present in the plants components including seeds, flowers, stem and leaves and protects skin from ultraviolet ray and chemicals. Novel study was performed using human melanoma cells and basal cell carcinoma cells to explain the effects of ingredients of garlic including diallyl sulfide, diallyl disulfide, and diallyl trisulfide. It was reported that diallyl trisulfide showed better growth inhibition of cancer cells and basal cell carcinoma cells than diallyl disulfide and diallyl sulfide. Moreover, diallyl trisulfide also exhibited selective target of growth inhibition between skin cancer cells and normal keratinocyte cells (91). Earlier study was made to evaluate the prophylactic effect of diallyl disulfide in chemically induced mouse skin carcinogenesis. Result confirmed that diallyl disulfide dose-dependently reduced skin tumor incidence and multiplicity, which was linked to the up-regulation of antioxidant enzymes activities and the nuclear accumulation of Nrf2 (92). Inhibitory effects of diallyl trisulfide on activator protein 1 activation and cyclooxygenase-2 expression via modulation of JNK or Akt signaling may partly account for its antitumorpromoting effect on skin carcinogenesis (93). Topical application of diallyl sulfide or diallyl disulfide considerably inhibited skin papilloma formation and meaningfully increased the rate of survival in the murine model (94). Diallyl sulfide is a potential chemopreventive agent capable of controlling and regulating the tumor suppressor p53 along with its downstream effective molecule, p21/waf1 (95). In the support of role of diallyl sulfide in carcinogenesis, experiment was performed to investigate the role of diallyl sulfide on modulation of multiple p53 and rasinduced signaling pathways in chemical induced skin carcinogenesis. Finding revealed that diallyl sulfide showed pivotal role in the upregulation of expression of tumor suppressor protein p53 and its downstream target molecule p21/waf1 and bax was upregulated by diallyl sulfide (96). Garlic extract was prepared and cytotoxic activities on melanoma cell line was examined. Finding revealed that that garlic extract induced a significant cytotoxic activity on melanoma cell line (97).

\subsection{Thyroid cancer}

Yangfeng Xiang and colleague performed study on thyroid cancer to evaluate the role of allicin. Result exhibited that combination of allicin and cisplatin or carboplatin caused enhanced growth inhibitory effect on cancer cells. Also, treatment with allicin significantly increased cancer cell autophagy. In addi- 
tion, western blot analysis based result showed that allicin treatment inhibited the activation of Akt, mammalian target of rapamycin and S6 (98). Anaplastic thyroid carcinoma-based study was performed and result confirmed that diallyl sulfide treatment increased the accumulation of sub-G1 DNA and concomitant accumulation of cells in the G2/M phase in a dose-dependent manner. Moreover, diallyl sulfide -induced apoptosis was linked with a decrease in the level of $\mathrm{Bcl}-2$ expression and an increase in the level of Bax expression (99). In this regard, earlier finding reported that diallyl trisulfide exerted an apoptosis-inducing effect on papillary-thyroid-cancer cells through activation of the MAPK signaling pathway (100).

\subsection{Urinary bladder cancer}

Induction of apoptosis by diallyl trisulfide in human bladder cancer cells based on in vitro was examined. Treatment of cancer cells with diallyl trisulfide resulted in potent anti-proliferative activity. In addition, diallyl trisulfide showed role in the reduction of the expression of anti-apoptotic Bcl-2 and Bcl-xL, and whereas the expression of pro-apoptotic Bax and death receptor-related proteins was increased (101). Bladder cancer cells based study was performed to evaluate the role of diallyl trisulfide it was reported that diallyl trisulfide suppressed proliferation of bladder cancer cells in a dose- and time-dependent manner which was linked with induced G2/M Phase cell cycle arrest and apoptosis and, diallyl trisulfide inhibits phosphatidylinositol 3'-kinase/Akt activation (102). Another study result concluded that allicin, an active compound of garlic has a noticeable tumor inhibitory effect on bladder tumor (103).

\section{Conclusion}

Cancer is a multifactorial disease characterized by alterations in biological process and cell signaling pathways. Cancer leads to devastating complications and the death of millions of people in the world every year. The mortality of cancer is rapidly increasing and estimated millions of deaths every year. The exact explanations behind this are complex but it is considered that various factors including alcohol consumption, cigarette smoking, HPV infection and various unknown factor play role in this regard. The current mode of treatment including chemotherapy, surgery and radiotherapy are effective, but millions of patients do not have access to treatment because current mode of treatment is often expensive, unavailable and causes adverse effects on normal cells. The most imperative difficult in the treatment of cancer is killing of tumor cells, without damaging/ altering the normal cells structure and functions. Natural therapies, including plant-derived products in management of cancer, may reduce adverse side effects and cost of treatment. The plant-derived products are rich resources of antioxidant which were found to be useful in the management of cancer. In this context, garlic contains various bioactive compound including allicin, diallyl sulfide, diallyl disulfide and such compounds has strong antioxidant property. Moreover, antioxidants property of active compound of garlic act as free radical hunters through inhibiting the damages caused by reactive oxygen species, and finally inhibit the pathogenesis of cancer. Several experimental studies based on in vivo and in vitro have confirmed garlic role as anti-proliferative in multiple cancer via growth inhibition and killing of cancer cells properties. Moreover, Garlic combination with cancer drugs are gaining popularity in the cancer management. The current review gathered the detail information of garlic and its bioactive compound role in various types of cancer cells through anti-proliferative and anti-cancerous effects. More studies are therefore needed to evaluate the toxicological evidence, mechanism and mode of action of garlic and its bioactive compound in the treatment and prevention of cancer and to develop the therapeutic strategies. Moreover, further preclinical and clinical studies are needed to validate the potentiality of bioactive compound of garlic.

\section{Funding}

None

\section{Conflict of interest}

The author declares no conflict of interest, financial or otherwise.

\section{Data availability}

The author declares the data supporting the findings of this study are available within the article.

\section{Acknowledgement}

I thank the College of Applied Medical Sciences, Qassim University for providing internet facility and access to journals to complete this study. 


\section{References}

1. Vissink A, Jansma J, Spijkervet FKL, et al. OralSequelae ofHead andNeckRadiotherapy. Critical Reviews in Oral Biology \& Medicine. 2003; 14(3): 199212. Available from: https://dx.doi.org/10.1177/ 154411130301400305.

2. Hong YS. Effects of allyl sulfur compounds and garlic extract on the expression of Bcl-2, Bax, and p53 in non small cell lung cancer cell lines. Experimental \& Molecular Medicine. 2000; 32(3): 127-134.

3. Farhadi F. Garlic ((Allium sativum)) Fresh Juice Induces Apoptosis in Human Oral Squamous Cell Carcinoma: The Involvement of Caspase-3, Bax and Bcl-2. J Dent Res Dent Clin Dent Prospects. 2015; 9(4): 267-73.

4. Dong M. Aged black garlic extract inhibits HT29 colon cancer cell growth via the PI3K/Akt signaling pathway. 2014; 2: 250-254.

5. JIKIHARA H, QI G, NOZOE K, et al. Aged garlic extract inhibits 1,2-dimethylhydrazine-induced colon tumor development by suppressing cell proliferation. Oncology Reports. 2015; 33(3): 1131-1140. Available from: https://dx.doi.org/10.3892/or.2014.3705.

6. Mousa AS, Mousa SA. Anti-Angiogenesis Efficacy of the Garlic Ingredient Alliin and Antioxidants: Role of Nitric Oxide and p53. Nutrition and Cancer. 2005; 53(1): 104-110. Available from: https://dx.doi.org/10. 1207/s15327914nc5301_12.

7. Liao QJ. Effect of diallyl disulfide on cell cycle arrest of human colon cancer SW480 cells. Ai Zheng. 2009; 28(2): 138-141.

8. Stiles B. PTENless means more. Developmental biology. 2004; 273(2): 175-184.

9. Downes CP. Stimulation of PI 3-kinase signaling via inhibition of the tumor suppressor phosphatase, PTEN Advances in enzyme regulation. 2007; 1: 184-194.

10. Zhang Y. Garlic-derived compound Sallylmercaptocysteine inhibits cell growth and induces apoptosis via the JNK and p38 pathways in human colorectal carcinoma cells. Oncology letters. 2014; 8(6): 2591-2596.

11. Lee CG. Allicin inhibits invasion and migration of breast cancer cells through the suppression of VCAM1: Regulation of association between p65 and ER- $\alpha$. Journal of functional foods. 2015; 15: 172-185.

12. Tsubura A. Anticancer effects of garlic and garlic-derived compounds for breast cancer control Anti-Cancer Agents in Medicinal Chemistry (Formerly Current Medicinal Chemistry-Anti-Cancer Agents). 2011; 11: 249-253.

13. Lei $X Y$. Apoptosis induced by diallyl disulfide in human breast cancer cell line MCF-7 1. Acta pharmacologica Sinica. 2008; 29(10): 1233-1239.

14. Nakagawa H. Growth inhibitory effects of diallyl disulfide on human breast cancer cell lines. Carcinogenesis. 2001; 22(6): 891-897. Available from: https://dx. doi.org/10.1093/carcin/22.6.891.
15. Altonsy MO, Habib TN, Andrews SC. Diallyl DisulfideInduced Apoptosis in a Breast-Cancer Cell Line (MCF-7) May Be Caused by Inhibition of Histone Deacetylation. Nutrition and Cancer. 2012; 64(8): 1251-1260. Available from: https://dx.doi.org/10. 1080/01635581.2012.721156.

16. Na HK. Diallyl trisulfide induces apoptosis in human breast cancer cells through ROS-mediated activation of JNK and AP-1. Biochemical pharmacology. 2012; 84(10): 1241-1250.

17. Xiao $X$. Diallyl disulfide suppresses SRC/Ras/ERK signaling-mediated proliferation and metastasis in human breast cancer by up-regulating miR-34a. PLoS One. 2014; 9(11): 112720-112720.

18. Huang J. Diallyl disulfide inhibits growth and metastatic potential of human triple-negative breast cancer cells through inactivation of the $\beta$-catenin signaling pathway Molecular nutrition \& food research. 2015; 59: 10631075.

19. Li X. Diallyl Trisulfide inhibits breast cancer stem cells via suppression of Wnt/ $\beta$-catenin pathway. Journal of cellular biochemistry. 2018; 119(5): 4134-4141.

20. $\mathrm{Hu} X$. Induction of glutathione S-transferase $\pi$ as a bioassay for the evaluation of potency of inhibitors of benzo (a) pyrene-induced cancer in a murine model. International journal of cancer. 1997; 73(6): 897-902.

21. Li C. Allicin induces apoptosis through activation of both intrinsic and extrinsic pathways in glioma cells. Molecular medicine reports. 2018; 17(4): 5976-5981.

22. Cha JH. Allicin inhibits cell growth and induces apoptosis in U87MG human glioblastoma cells through an ERK-dependent pathway. Oncology reports. 2012; 28(1): 41-48.

23. Gao XY. Effect of combined treatment with cyclophosphamidum and allicin on neuroblastoma-bearing mice Asian Pacific journal of tropical medicine. 2015; 8: 137141.

24. Welch C, Wuarin L, Sidell N. Antiproliferative effect of the garlic compound S-allyl cysteine on human neuroblastoma cells in vitro. Elsevier BV, 1992. Available from: https://dx.doi.org/10.1016/0304-3835(92) 90263-u.

25. Filomeni G. Reactive oxygen species-dependent c-Jun NH2-terminal kinase/c-Jun signaling cascade mediates neuroblastoma cell death induced by diallyl disulfide Cancer research. 2003; 63: 5940-5949.

26. Tao Q. Diallyl trisulfide inhibits proliferation, invasion and angiogenesis of glioma cells by inactivating Wnt/ $\beta$ catenin signaling Cell and tissue research. 2017; 370: 379-390.

27. Li Y. Diallyl trisulfide inhibits proliferation, invasion and angiogenesis of osteosarcoma cells by switching on suppressor microRNAs and inactivating of Notch-1 signaling. Carcinogenesis. 2013; 34(7): 1601-1611.

28. Li Y. Diallyl disulfide suppresses FOXM1-mediated proliferation and invasion in osteosarcoma by upregulating miR-134. J Cell Biochem. 2018; . 
29. Xie WP. Treatment of Saos-2 osteosarcoma cells with diallyl trisulfide is associated with an increase in calreticulin expression. Exp Ther Med. 2018; 15(6): 4737-4742.

30. Wang $\mathrm{H}$. Diallyl trisulfide induces osteosarcoma cell apoptosis through reactive oxygen species-mediated downregulation of the PI3K/Akt pathway. Oncol Rep. 2016; 35(6): 3648-58.

31. Jiang $W$. The synergistic anticancer effect of artesunate combined with allicin in osteosarcoma cell line in vitro and in vivo. Asian Pac J Cancer Prev. 2013; 14(8): 4615-4624.

32. Wu PP. Diallyl sulfide induces cell cycle arrest and apoptosis in HeLa human cervical cancer cells through the p53, caspase-and mitochondriadependent pathways. International Journal of Oncology. 2011 ; 38(6): 1605-1613.

33. Lin YT. Diallyl disulfide (DADS) induces apoptosis in human cervical cancer Ca Ski cells via reactive oxygen species and Ca2+-dependent mitochondriadependent pathway. Anticancer research. 2008; 28(5A): 2791-2799.

34. Di C. Diallyl disulfide enhances carbon ion beamsinduced apoptotic cell death in cervical cancer cells through regulating Tap73 /DeltaNp73. Cell Cycle. 2015; 14(23): 3725-3758.

35. Zhang Q, Yang D. Allicin suppresses the migration and invasion in cervical cancer cells mainly by inhibiting NRF2. Exp Ther Med. 2019; 17(3): 1523-1528.

36. Shirin H. Antiproliferative effects of $S$ allylmercaptocysteine on colon cancer cells when tested alone or in combination with sulindac sulfide Cancer research. 2001; 61: 725-731.

37. Yang JS. Diallyl disulfide induces apoptosis in human colon cancer cell line (COLO 205) through the induction of reactive oxygen species, endoplasmic reticulum stress, caspases casade and mitochondrialdependent pathways. Food and Chemical Toxicology. 2009; 47(1): 171-179.

38. Hosono T. Diallyl trisulfide suppresses the proliferation and induces apoptosis of human colon cancer cells through oxidative modification of $\beta$-tubulin. Journal of Biological Chemistry. 2005; 280(50): 4148741493.

39. Huang YS. Diallyl disulfide inhibits the proliferation of HT-29 human colon cancer cells by inducing differentially expressed genes. Molecular medicine reports. 2011; 4(3): 553-559.

40. Yu CS. Diallyl trisulfide induces apoptosis in human primary colorectal cancer cells. Oncology reports. 2012; 28(3): 949-954.

41. Zhang Q. Wnt/ $\beta$-catenin signaling mediates the suppressive effects of diallyl trisulfide on colorectal cancer stem cells Cancer chemotherapy and pharmacology. 2018; 81: 969-977.

42. Wargovich MJ. Diallyl sulfide, a flavor component of garlic (Allium sativum), inhibits dimethyihydrazine- induced colon cancer. Carcinogenesis. 1987; 8(3): 487-489. Available from: https://dx.doi.org/10.1093/ carcin/8.3.487.

43. Li N. A proteomic investigation into a human gastric cancer cell line BGC823 treated with diallyl trisulfide. Carcinogenesis. 2006; 27(6): 1222-1231.

44. Su B. Identification of potential targets for diallyl disulfide in human gastric cancer MGC-803 cells using proteomics approaches. Oncology reports. 2015; 33(5): 2484-2494.

45. Pan Y. Epigenetic upregulation of metallothionein $2 \mathrm{~A}$ by diallyl trisulfide enhances chemosensitivity of human gastric cancer cells to docetaxel through attenuating NF- $\kappa$ B activation. Antioxidants \& redox signaling. 2016; 24(15): 839-854.

46. Jiang $X Y$. Diallyl trisulfide suppresses tumor growth through the attenuation of Nrf2/Akt and activation of p38/JNK and potentiates cisplatin efficacy in gastric cancer treatment. Acta Pharmacologica Sinica. 2017; 38(7): 1048-1048.

47. Jiang $X$. Diallyl trisulfide inhibits growth of $\mathrm{NCl}-\mathrm{H} 460$ in vitro and in vivo, and ameliorates cisplatin-induced oxidative injury in the treatment of lung carcinoma in xenograft mice. International journal of biological sciences. 2017; 13(2): 167-167.

48. Ling $\mathrm{H}$. Erk is involved in the differentiation induced by diallyl disulfide in the human gastric cancer cell line MGC803 Cellular \& molecular biology letters. 2006; 11 : 408-408.

49. Sun L, Wang $X$. Effects of allicin on both telomerase activity and apoptosis in gastric cancer SGC-7901 cells. World journal of gastroenterology. 2003; 9(9): 1930-1930.

50. Zhang X. Allicin induces apoptosis of the MGC-803 human gastric carcinoma cell line through the p38 mitogen-activated protein kinase/caspase-3 signaling pathway Molecular medicine reports. 2015; 11: 27552760.

51. Song B. Allicin inhibits human renal clear cell carcinoma progression via suppressing HIF pathway International journal of clinical and experimental medicine. 2015; 8: 20573-20573.

52. Chu YL. Allicin induces anti-human liver cancer cells through the p53 gene modulating apoptosis and autophagy. Journal of agricultural and food chemistry. 2013; 61(41): 9839-9848.

53. Zou $X$. Allicin sensitizes hepatocellular cancer cells to anti-tumor activity of 5-fluorouracil through ROS-mediated mitochondrial pathway. Journal of Pharmacological Sciences. 2016; 131(4): 233-240.

54. Wu CC. Differential effects of allyl sulfides from garlic essential oil on cell cycle regulation in human liver tumor cells. Food and Chemical Toxicology. 2004; 42(12): 1937-1947.

55. Yu FS. Diallyl sulfide inhibits murine WEHI-3 leukemia cells in BALB/c mice in vitro and in vivo. Human \& 
experimental toxicology. 2009; 28(12): 785-790.

56. Hung FM. Effects of diallyl trisulfide on induction of apoptotic death in murine leukemia WEHI-3 cells in vitro and alterations of the immune responses in normal and leukemic mice in vivo. Environmental toxicology. 2015; 30(11): 1343-1353.

57. Tan $\mathrm{H}$. Inhibition of ERK and activation of $p 38$ are involved in diallyl disulfide induced apoptosis of leukemia HL-60 cells Archives of pharmacal research. 2008; 31: 786-786.

58. Suda S. Identification of molecular target of diallyl trisulfide in leukemic cells Bioscience, biotechnology, and biochemistry. 2014; 78: 1415-1417.

59. Choi YH, Park HS. Apoptosis induction of U937 human leukemia cells by diallyl trisulfide induces through generation of reactive oxygen species. Journal of biomedical science. 2012; 19(1): 50-50.

60. Dirsch VM, Antlsperger DSM, Hentze $H$, et al. Ajoene, an experimental anti-leukemic drug: mechanism of cell death. Leukemia. 2002; 16(1): 74-83. Available from: https://dx.doi.org/10.1038/sj.leu.2402337.

61. Huang L. Allicin inhibits the invasion of lung adenocarcinoma cells by altering tissue inhibitor of metalloproteinase/matrix metalloproteinase balance via reducing the activity of phosphoinositide 3-kinase/AKT signaling Oncology letters. 2017; 14: 468-474.

62. Xiao D. Diallyl trisulfide selectively causes Bax-and Bak-mediated apoptosis in human lung cancer cells Environmental and molecular mutagenesis. 2009; 50: 201-212.

63. Li W. Diallyl trisulfide induces apoptosis and inhibits proliferation of A549 cells in vitro and in vivo. Acta Biochim Biophys Sin. 2012; 44(7): 577-583.

64. Jin ZY. Raw garlic consumption as a protective factor for lung cancer, a population-based case-control study in a Chinese population. Cancer prevention research. 2013; (6): 711-718.

65. Myneni AA. Raw garlic consumption and lung cancer in a Chinese population. Cancer Epidemiology and Prevention Biomarkers. 2016; 25(4): 624-633.

66. Tang FY, Chiang EP, Pai MH. Consumption ofSAllylcysteine Inhibits the Growth of Human NonSmall-Cell Lung Carcinoma in a Mouse Xenograft Model. Journal of Agricultural and Food Chemistry. 2010; 58(20): 11156-11164. Available from: https: //dx.doi.org/10.1021/jf102539k.

67. Dasgupta P, (Bandyopadhyay) SS. Role of diallyl disulfide-mediated cleavage of c-Myc and Sp-1 in the regulation of telomerase activity in human lymphoma cell line U937. Elsevier BV, 2015. Available from: https://dx.doi.org/10.1016/j.nut.2015.02.016.

68. Shigemi Z. Diallyl trisulfide induces apoptosis by suppressing NF- $k B$ signaling through destabilization of TRAF6 in primary effusion lymphoma. International journal of oncology. 2016; 48(1): 293-304.

69. Rahmani AH. Curcumin: a potential candidate in prevention of cancer via modulation of molecular pathways. 2014; .

70. Rahmani AH. Implications of green tea and its constituents in the prevention of cancer via the modulation of cell signaling pathway. BioMed Research International. 2015; .

71. Rahmani AH. Therapeutic effects of date fruits (Phoenix dactylifera) in the prevention of diseases via modulation of anti-inflammatory, anti-oxidant and anti-tumor activity. International journal of clinical and experimental medicine. 2014; 7(3): 483-483.

72. Rahmani A, Alsahli M, Almatroodi S. Potential antitumor effects of pomegranates and its ingredients. Medknow, 2017. Available from: https://dx.doi.org/ 10.4103/phrev.phrev_25_17.

73. Almatroudi A, Alsahli MA, Alrumaihi F, et al.. Ginger: A Novel Strategy to Battle Cancer through Modulating Cell Signalling Pathways: A Review. Bentham Science Publishers Ltd., 2019. Available from: https://dx.doi. org/10.2174/1389201020666190119142331.

74. Pai MH. S-Allylcysteine inhibits tumor progression and the epithelial-mesenchymal transition in a mouse xenograft model of oral cancer. British Journal of Nutrition. 2012; 108(1): 28-38.

75. Xu YS. S-allylcysteine, a garlic derivative, suppresses proliferation and induces apoptosis in human ovarian cancer cells in vitro. Acta Pharmacol Sin. 2014; 35(2): 267-74.

76. Xu Y. S-allylcysteine suppresses ovarian cancer cell proliferation by DNA methylation through DNMT1. J Ovarian Res. 2018; 11(1): 39-39.

77. Wan HF. Effect of diallyl trisulfide on human ovarian cancer SKOV- 3/DDP cell apoptosis. Asian Pac J Cancer Prev. 2013; 14(12): 7197-201.

78. $\mathrm{Xu} \mathrm{L}$. Role of JNK activation and mitochondrial Bax translocation in allicin-induced apoptosis in human ovarian cancer SKOV3 cells Evidence-Based Complementary and Alternative Medicine. 2014; .

79. Wang CJ. Effect of combined treatment with recombinant interleukin-2 and allicin on pancreatic cancer. Mol Biol Rep. 2013; 40(12): 6579-85.

80. Ma HB. Apoptotic pathway induced by diallyl trisulfide in pancreatic cancer cells. World Journal of Gastroenterology. 2014; 20(1): 193-193.

81. Stan S. Garlic-derived organosulfur compound diallyl trisulfide induces apoptosis in pancreatic cancer cells. 2013; .

82. Arunkumar A. Growth suppressing effect of garlic compound diallyl disulfide on prostate cancer cell line (PC-3) in vitro. Biological and Pharmaceutical Bulletin. 2005; 28(4): 740-743.

83. Arunkumar A. Garlic compound, diallyl disulfide induces cell cycle arrest in prostate cancer cell line PC3 Molecular and cellular biochemistry. 2006; 288: 107113.

84. Stan SD, Singh SV. Transcriptional Repression and Inhibition of Nuclear Translocation of Androgen 
Receptor by Diallyl Trisulfide in Human Prostate Cancer Cells. Clinical Cancer Research. 2009; 15(15): 4895-4903. Available from: https://dx.doi.org/10. 1158/1078-0432.ccr-09-0512.

85. Gunadharini DN, Arunkumar A, Krishnamoorthy G, et al. Antiproliferative effect of diallyl disulfide (DADS) on prostate cancer cell line LNCaP. Cell Biochemistry and Function. 2006; 24(5): 407-412. Available from: https://dx.doi.org/10.1002/cbf.1262.

86. Xiao D. Diallyl trisulfide-induced apoptosis in human prostate cancer cells involves c-Jun N-terminal kinase and extracellular-signal regulated kinase-mediated phosphorylation of Bcl-2. Oncogene. 2004; 23(33): 5594-5594.

87. Kim YA. Mitochondria-mediated apoptosis by diallyl trisulfide in human prostate cancer cells is associated with generation of reactive oxygen species and regulated by Bax/Bak Molecular cancer therapeutics. 2007; 6: 1599-1609.

88. Xiao D, Singh SV. Diallyl trisulfide, a constituent of processed garlic, inactivates Akt to trigger mitochondrial translocation of BAD and caspase-mediated apoptosis in human prostate cancer cells. Carcinogenesis. 2006; 27(3): 533-540. Available from: https://dx.doi. org/10.1093/carcin/bgi228.

89. Borkowska A, Knap N, Antosiewicz J. Diallyl Trisulfide Is More Cytotoxic to Prostate Cancer Cells PC-3 than to Noncancerous Epithelial Cell Line PNT1A: A Possible Role of p66Shc signaling Axis. Nutrition and Cancer. 2013; 65(5): 711-717. Available from: https: //dx.doi.org/10.1080/01635581.2013.789115.

90. Howard EW. Garlic-derived S-allylmercaptocysteine is a novel in vivo antimetastatic agent for androgenindependent prostate cancer Clinical Cancer Research. 2007; 13: 1847-1856.

91. Wang HC. Allyl sulfides inhibit cell growth of skin cancer cells through induction of DNA damage mediated G2/M arrest and apoptosis. Journal of agricultural and food chemistry. 2010; 58(11): 70967103.

92. Shan Y. Prophylaxis of diallyl disulfide on skin carcinogenic model via p21-dependent Nrf2 stabilization. Scientific reports. 2016; 6: 35676-35676.
93. Shrotriya S. Diallyl Trisulfide Inhibits Phorbol EsterInduced Tumor Promotion, Activation of AP-1, and Expression of COX-2 in Mouse Skin by Blocking JNK and Akt Signaling Cancer research. 2010; 70: 1932-1940.

94. Dwivedi C. Chemoprevention of chemically induced skin tumor development by diallyl sulfide and diallyl disulfide. Pharmaceutical research. 1992; 9(12): 16681670.

95. Arora A, Siddiqui IA, Shukla Y. Modulation of p53 in 7, 12-dimethylbenz [a] anthracene-induced skin tumors by diallyl sulfide in Swiss albino mice. Molecular Cancer Therapeutics. 2004; 3(11): 1459-1466.

96. Kalra N, Arora A, Shukla Y. Involvement of multiple signaling pathways in diallyl sulfide mediated apoptosis in mouse skin tumors. 2006; .

97. Hakimzadeh H, Ghazanfari T, Rahmati B, et al. Cytotoxic effect of garlic extract and its fractions on Sk-mel3 melanoma cell line. Immunopharmacology and Immunotoxicology. 2010; 32(3): 371375. Available from: https://dx.doi.org/10.3109/ 08923970903420574.

98. Xiang Y. Allicin activates autophagic cell death to alleviate the malignant development of thyroid cancer Experimental and therapeutic medicine. 2018; 15: 3537-3543.

99. Shin HA. Diallyl sulfide induces growth inhibition and apoptosis of anaplastic thyroid cancer cells by mitochondrial signaling pathway. Oral oncology. 2010; 46(4): 15-18.

100. Pan J. Induction of Apoptosis in Human PapillaryThyroid-Carcinoma BCPAP Cells by Diallyl Trisulfide through Activation of the MAPK Signaling Pathway. Journal of agricultural and food chemistry. 2018; 66(23): 5871-5878.

101. Shin DY. Diallyl trisulfide-induced apoptosis of bladder cancer cells is caspase-dependent and regulated by PI3K/Akt and JNK pathways Environmental toxicology and pharmacology. 2014; 37: 74-83.

102. Wang YB. Diallyl trisulfide induces $\mathrm{Bcl}-2$ and caspase3-dependent apoptosis via downregulation of Akt phosphorylation in human T24 bladder cancer cells. Phytomedicine. 2010; 17(5): 363-368.

103. Jian W. Effect of Allicin in antagonizing mice's bladder cancer in vitro and in vivo. Chinese Journal of Integrative Medicine. 2004; 10(3): 208-212. 